\section{$\underset{\substack{\text { hommes } \\ \text { \& migrations }}}{ }$}

\section{Hommes \& migrations}

Revue française de référence sur les dynamiques

migratoires

$1298 \mid 2012$

France - Algérie, le temps du renouveau

\title{
Bilan et perspectives des migrations algériennes
}

\section{Kamel Kateb}

\section{(2) OpenEdition}

Journals

\section{Édition électronique}

URL : http://journals.openedition.org/hommesmigrations/1568

DOI : 10.4000/hommesmigrations. 1568

ISSN : 2262-3353

\section{Éditeur}

Musée national de l'histoire de l'immigration

\section{Édition imprimée}

Date de publication : 1 juillet 2012

Pagination : 6-21

ISSN : 1142-852X

Référence électronique

Kamel Kateb, «Bilan et perspectives des migrations algériennes », Hommes \& migrations [En ligne], 1298 | 2012, mis en ligne le 31 décembre 2014, consulté le 19 avril 2019. URL : http://

journals.openedition.org/hommesmigrations/1568; DOI : 10.4000/hommesmigrations. 1568 


\section{Bilan et perspectives des migrations algériennes}

Par Kamel Kateb, chercheur démographe à l'Ined(")

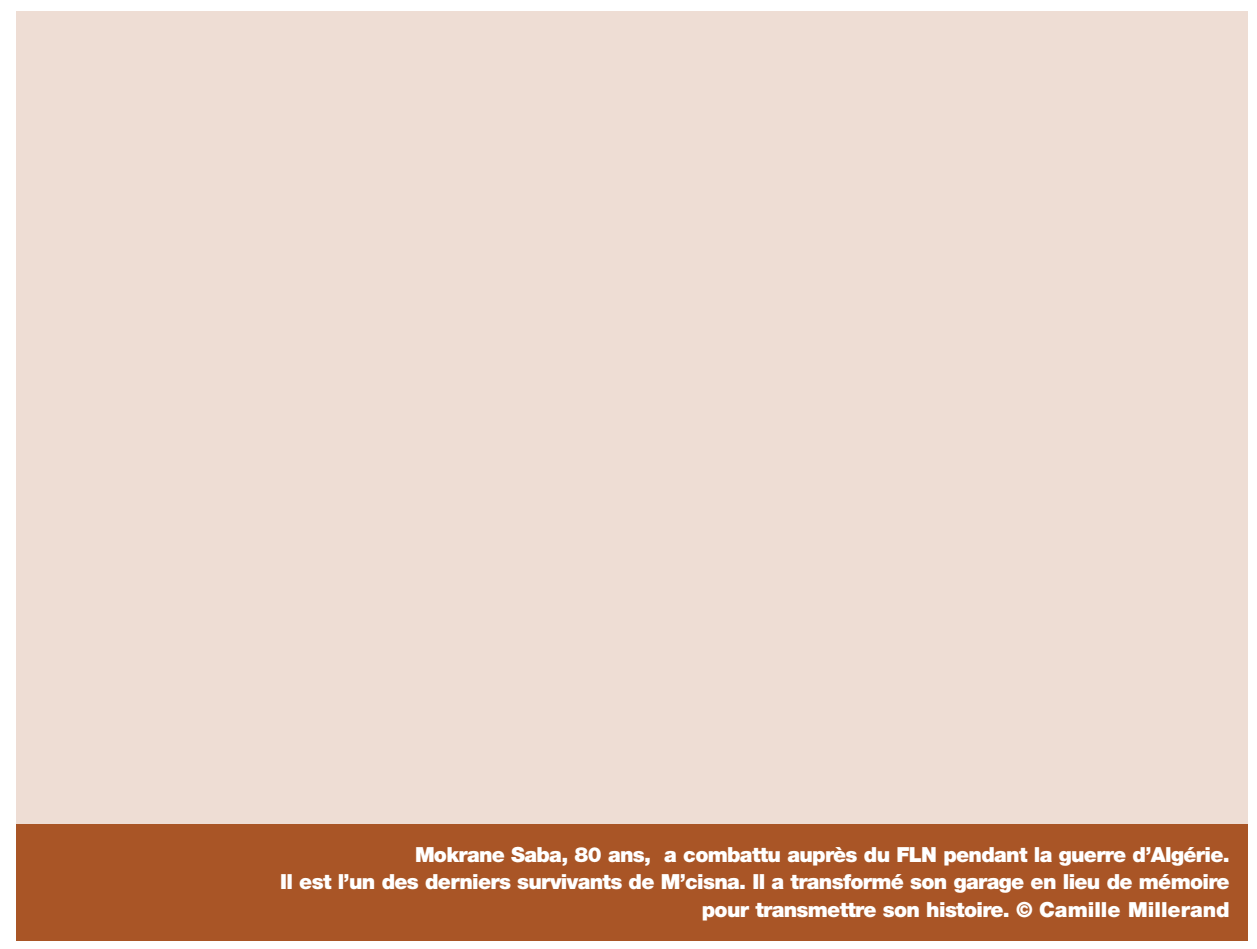

Pays d'immigration pour les Européens durant la période coloniale, l'Algérie est depuis plus d'un demi-siècle un pays d'émigration massive. Aujourd'hui, de nouvelles tendances se font jour dans l'émigration des Algériens en direction des pays européens et notamment de la France.

Le profil des migrants a évolué en fonction de ces changements : l'émigration se féminise, les migrants sont jeunes et davantage instruits De quoi pallier les déficits démographiques que l'Europe ne va pas manquer de connaître dans les prochaines années. 
Situés au sud du Bassin méditerranéen, les pays du Maghreb et plus particulièrement l'Algérie ont connu depuis l'Antiquité de grands mouvements de population. Pendant des siècles, ils ont été principalement des pays d'immigration. Il a fallu attendre la deuxième moitié du $\mathrm{XX}^{\mathrm{e}}$ siècle pour assister à une inversion durable des flux migratoires et voir la région se transformer en zone d'émigration. Au début du $\mathrm{XX}^{\mathrm{e}}$ siècle, les immigrés européens en Algérie ont représenté près de $15 \%$ de la population totale et plus de $10 \%$ dans l'ensemble de la région du Maghreb. À la veille des indépendances, au début des années cinquante, ils ne sont plus que 10,4 \% de la population résidant en Algérie et $8 \%$ de celle du Maghreb (tableau 1). Cette évolution est le fait du début de l'inversion des flux entre les deux rives de la Méditerranée et d'une fécondité différentielle considérable entre le Nord et le Sud. La décolonisation a accéléré un processus qui se dessinait déjà au lendemain de la Seconde Guerre mondiale. L'émigration des Algériens vers la France a démarré à la veille de la Première Guerre mondiale en lien avec le contexte historique de l'époque. Un petit nombre seulement de migrants de cette période se sont installés définitivement en France ${ }^{(2)}$. La crise économique de 1929-1933 et les prémices de la Seconde Guerre mondiale avaient provoqué une migration de retour. Cette émigration ne s'est installée durablement en France que dans la seconde moitié du XX $\mathrm{XX}^{\mathrm{e}}$ siècle. Elle a coïncidé avec l'accession à l'indépendance des pays de la région. De pays d'immigration, ils se sont transformés en pays d'émigration en moins d'une dizaine d'années. Leur taux d'émigration a été multiplié par trois en cinquante ans ${ }^{(3)}$.

\section{Avec la conquête, des Algériens partent, des Européens arrivent}

La conquête de l'Algérie a généré des flux migratoires importants : l'arrivée d'une population européenne et le départ vers les pays musulmans des Algériens qui refusaient la domination d'un gouvernement d'une religion différente. Le gouvernement français, d'abord favorable à cet exode de tribus hostiles à la présence française, fut vite embarrassé par le caractère politico-religieux d'un tel mouvement d'émigration ${ }^{(4)}$. Ces flux migratoires vers les pays voisins et le Moyen-Orient, la Syrie en particulier, ont perduré tout le long du XIX et au début du XX $\mathrm{XX}^{\mathrm{e}}$ siècle.

La conquête, en 1830, a été marquée par l'expulsion d'Alger de 10000 Turcs qui ont été embarqués manu militari vers la ville turque de Smyrne. À ces expulsés, il faudrait ajouter le départ volontaire d'“indigènes" dont la comptabilité ne pourra probablement jamais être faite : ces départs étaient directement liés à la guerre de conquête, jusqu'à la défaite d'Abdelkader en 1847. Le bannissement des chefs de 
guerre, de leurs familles et des groupes d'individus considérés comme dangereux pour l'ordre public a ensuite été prononcé en raison de leur implication dans les mouvements de résistance à la colonisation. Ils ont été internés à Saint-Ambroise (pour l'émir et ses proches), en Corse, dans l'île de Sainte-Marguerite, dans les Antilles françaises et en Nouvelle-Calédonie pour les responsables de l'insurrection de $1871^{(5)}$. Les expulsions et les déportations ont été suivies par des départs volontaires individuels ou collectifs vers le Maroc et la Tunisie, puis vers le Moyen-Orient où un pays, la Syrie, a attiré la majorité des migrants.

Compte tenu des données statistiques disponibles, il est impossible de prendre toute la mesure de cette émigration. Les différents recensements du XIX ${ }^{\mathrm{e}}$ siècle s'appliquaient à des territoires de superficies variables, et une partie seulement des "indigènes" était dénombrée sommairement. De même, l'état civil n'a été constitué pour l'Algérie du Nord qu'à la fin du XIX ${ }^{e}$ siècle. On peut toutefois donner un ordre de grandeur de ces mouvements migratoires entre les recensements de 1911 et 1921. La différence entre la population observée et la population attendue - -313 400 donnerait un ordre de grandeur de l'émigration. Cependant, cette période a aussi été marquée par une émigration vers la France. La mobilisation des Algériens dans l'armée française et la réquisition de travailleurs pour les usines de métropole ont accéléré les migrations vers la France. À la veille de la Première Guerre mondiale, les archives françaises signalent la présence en France de 5000 Algériens. En 1924, ces mêmes services évaluent ce nombre à 100 000. Si nous le retenons pour 1921, ce seraient alors plus de 200000 Algériens qui, pendant la décennie 1911-1921, auraient quitté leur pays pour une autre destination que la France : le Moyen-Orient. Ce nombre, calculé à partir des données statistiques disponibles, représente $4,2 \%$ de la population enregistrée durant le recensement de $1911^{(6)}$.

À l'inverse, les flux migratoires de l'Europe vers l'Algérie ont débuté dès la conquête d'Alger. Ils ont été le fait d'initiatives personnelles qui correspondaient à des flux migratoires au départ de l'Europe du Sud, ces migrants mettant à profit le nouvel espace ouvert par la colonisation de l'Algérie. Les crises politiques, économiques et sociales (les révolutions, les défaites militaires et la propagation du phylloxéra à la fin du siècle, etc.) ont crée des opportunités pour le développement du peuplement européen ${ }^{(7)}$. Mais il correspond aussi à la volonté politique affirmée de substituer à la population indigène d'Algérie une population européenne ${ }^{(8)}$. Durant tout le $\mathrm{XIX}^{\mathrm{e}}$ siècle, le Parlement français était sollicité pour soutenir financièrement le processus de colonisation et de peuplement de l'Algérie. Cette population a, au fil des ans, engendré une descendance qui n'avait pratiquement aucun lien avec le pays d'origine des parents ou des grands-parents (Italie, Espagne, Malte, etc.). Jusqu'à la Première Guerre mondiale, l'immigration constituait une part importante dans la 
Tableau 1 : Proportion dlimmigrés et d'émigrés dans la population totale (en \%)

\begin{tabular}{|c|c|c|c|c|c|c|c|c|}
\hline \multirow{2}{*}{$\begin{array}{c}\text { Pays } \\
\text { Années }\end{array}$} & \multicolumn{2}{|c|}{ Maghreb } & \multicolumn{2}{|c|}{ Algérie } & \multicolumn{2}{|c|}{ Maroc } & \multicolumn{2}{|c|}{ Tunisie } \\
\hline & Immigrés & Émigrés & Immigrés & Émigrés & Immigrés & Émigrés & Immigrés & Émigrés \\
\hline 1926 & 11 & 0,1 & 14,7 & 1,4 & 2,1 & 0,1 & 11,2 & 0 \\
\hline 1936 & 8,9 & 0,7 & 13,2 & 1,2 & 3,2 & 0,2 & 10,9 & 0 \\
\hline $\begin{array}{l}1950- \\
1956\end{array}$ & 8 & 1,2 & 10,4 & 2,5 & 4,5 & 0,1 & 9, & 0,1 \\
\hline $\begin{array}{c}1960- \\
1962\end{array}$ & & 1,5 & & 2,9 & & 0,3 & 1,5 & 0,6 \\
\hline $\begin{array}{l}1998- \\
2004\end{array}$ & 0,3 & 3,6 & 0,2 & 2,6 & 0,2 & 4,6 & 0,8 & 3,5 \\
\hline
\end{tabular}

Sources : Données reconstituées à partir des recensements des différents pays et des recensements français. Note : La proportion des immigrés est calculée à partir de la population immigrée et de ses descendants, celle des émigrés à partir des populations résidant à l'étranger sur la population indigène.

croissance totale de la population européenne, 57 \% entre 1896-1901, 40 \% de 1901 à 1906, et 48,2 \% de 1906 à 1911. La croissance naturelle devient, à partir de 1906, le principal facteur de croissance démographique et reste voisine de $1 \%$ par an en moyenne, d'autant que le courant migratoire se ralentit quelque peu. Le solde a été même négatif de 10804 personnes pendant la Première Guerre mondiale. En 1911, le recensement a fait ressortir que, sur 746000 Européens vivant en Algérie, 480000 d'entre eux étaient nés en Algérie, 113000 en France et 154000 dans les autres pays européens ${ }^{(9)}$.

\section{L'émigration algérienne, un bilan quantitatif difficile à cerner}

En l'absence de données statistiques mesurant l'émigration algérienne au départ de l'Algérie, l'analyse porte essentiellement sur les données enregistrées à l'entrée des pays de l'OCDE ${ }^{(11)}$. Les données relatives aux flux migratoires, fournies par les services de cette organisation internationale d'études économiques, montrent que près de 450000 Algériens ont été enregistrés entre 1995 et 2009 à l'entrée des pays formant cet ensemble de coopération entre pays industrialisés. Entre 2000 et 2009, plus de 360000 immigrants algériens y ont été enregistrés. Les entrées annuelles d'immigrants algériens (figure 1) représentent près de 40000 individus à la fin de la première décennie du $\mathrm{XXI}^{\mathrm{e}}$ siècle ; 55,5 \% d'entre elles ont concerné la France. Les 
entrées enregistrées à la fin des années quatre-vingt-dix et au début des années deux mille étaient de l'ordre de 20000 par an, dont 70 \% en France. Bien qu'en baisse en valeur relative, le nombre d'Algériens enregistrés à l'entrée du territoire français n'a cessé d'augmenter, un peu plus de 12000 en 1999 et en 2000, pour se situer à plus de 20000 en 2009, avec un maximum de 28000 en 2003. Cette augmentation des flux migratoires s'accompagne d'une diversification des destinations (figure 2): vers l'Espagne ( 8014 entrées en 2008 et 6376 en 2009), mais aussi vers le Canada (3 228 en 2008 et 4785 en 2009) et les États-Unis (1 485 en 2009).

\section{Tableau 2 : Immigrés maghrébins dans les pays de POCDE en 2005}

\begin{tabular}{|c|c|c|c|c|}
\hline Pays & Algérie & Maroc & Tunisie & Total \\
\hline Australie & 1245 & 1659 & 600 & 3504 \\
\hline Belgique & 20295 & 162626 & 10480 & 193401 \\
\hline Canada & 32250 & 39055 & 7405 & 78710 \\
\hline Danemark & 877 & 5067 & & 5944 \\
\hline Espagne & 58653 & 732006 & 252 & 790911 \\
\hline États-Unis & 12094 & 82342 & & 94436 \\
\hline Finlande & 595 & 1503 & 489 & 2587 \\
\hline France & 691361 & 633736 & 226684 & 1551781 \\
\hline Grèce & 320 & 802 & 294 & 1416 \\
\hline Hongrie & 633 & 109 & 88 & 830 \\
\hline Italie & 9985 & 147783 & 50647 & 208415 \\
\hline Luxembourg & 339 & 588 & 237 & 1164 \\
\hline Mexique & & 156 & 18 & 174 \\
\hline Nouvelle-Zélande & 123 & & 54 & 177 \\
\hline Norvège & 1077 & 4780 & 803 & 6660 \\
\hline Pays-Bas & 3904 & 166894 & 4283 & 175081 \\
\hline Pologne & & 167 & & 167 \\
\hline Royaume-Uni & 16000 & 20000 & & 36000 \\
\hline Slovaquie & 119 & 58 & & 177 \\
\hline Suède & 2333 & 6655 & 3695 & 12683 \\
\hline Suisse & 6013 & 9427 & 5713 & 21153 \\
\hline Total & 858216 & 2015413 & 311742 & 3185371 \\
\hline
\end{tabular}

Source: Données extraites des statistiques de l'OCDE, consultées le 5 janvier 2011. 
La volonté de blocage de l'immigration par les autorités européennes (et françaises, pour les Algériens) a finalement transformé les motifs déclarés de la migration. La migration pour motif de travail, principal moteur des phénomènes migratoires, disparaît presque totalement des déclarations. Dans la première décennie du $\mathrm{XXI}^{\mathrm{e}}$ siècle, le mariage est le principal motif d'introduction sur le territoire français (tableau 4). Ce motif concerne à la fois les ressortissants étrangers (migrants ayant conservé la nationalité algérienne sur le territoire français) et les Français (majoritairement des enfants d'immigrés algériens de nationalité française).

Entre les années 2000 et 2008, les entrées selon ce motif représentent 123566 personnes nées en Algérie enregistrées par le fichier de l'Application de gestion des dossiers des ressortissants étrangers en France (AGDREF) ${ }^{(12)}$, soit 62,4\% des entrées en provenance d'Algérie. Les conjoints de Français représentent 47,5 \% des entrées selon ce motif. La répartition selon le sexe est favorable au sexe féminin : 64734 entrées pour 61832 individus de sexe masculin.

Une autre composante de ces flux est constituée par les entrées sous visa "étudiant", de l'ordre de 3 à 4000 par an (figure 4). Elles sont estimées en France, selon les données fournies par les services de l'Office des migrations internationales (OMI), à 34000 entre 1995 et 2007. C'est objectivement une migration à caractère temporaire (pour la durée des études) mais qui tend à se transformer en migration définitive. Il faut préciser que, malgré la décision de 1974 d'arrêter l'immigration pour motif de travail, des centaines de personnes ont reçu chaque année des titres de séjour pour ce motif(figure 5). Pour la période 1995-2008, il y a eu 9693 admissions en tant que travailleurs.

\section{Quand la violence politique élargit le champ de la migration}

Une fraction relativement importante des flux annuels enregistrés est constituée par des demandeurs d'asile (figure 3). Les demandeurs d'asile algériens en Europe ont représenté 76675 personnes au cours de la période 1992-2008; ils étaient de l'ordre de quelques centaines dans la décennie précédente et ne concernaient que les Pays-Bas et les pays scandinaves ${ }^{(13)}$. Ils sont actuellement plusieurs centaines chaque année et s'adressent à un grand nombre de pays de l'Union européenne. Parmi eux, 25267 ont fait leur demande en France, soit près du tiers de l'ensemble des demandes d'asile en Europe. C'est le résultat de la détérioration des conditions de sécurité qui ont prévalu en Algérie depuis la fin de l'année 1991 et de l'arrêt du processus électoral qui avait donné la majorité aux islamistes. Il y a, par conséquent, un différentiel migratoire dont 
la cause directe ou indirecte serait le développement de la violence des groupes armés et de celle des appareils de sécurité de l'État algérien, auxquels s'ajoute probablement le climat d'insécurité produit par les émeutes en Kabylie et dans les autres régions de l'Algérie. Ces émeutes et leur caractère parfois insurrectionnel augmentent l'action répressive de l'État algérien (60 morts et plus de 900 blessés par balles en Kabylie au cours des émeutes du printemps 2001).

Devant les dispositifs de limitation de l'entrée aux frontières de l'Union européenne et les interprétations restrictives des conventions internationales concernant les réfugiés, une partie de la migration engendrée par la violence politique s'est probablement insérée dans les flux migratoires traditionnels (travail, études, regroupement familial, etc.). La migration qui s'est positionnée sur le terrain politique (demande d'asile, réfugiés politiques) a contribué à l'élargissement de l'espace migratoire des Algériens à une échelle européenne en multipliant les pays d'arrivée (les quelques dizaines de migrants qui s'installent dans les pays scandinaves le font le plus souvent à la faveur de procédures de demande d'asile). Ainsi, la violence politique, tenant compte des dispositifs existant à l'entrée des différents pays européens, a été un facteur d'accélération de la diversification des pays d'accueil pour les immigrés algériens.

À l'instar des autres courants migratoires, la féminisation de l'immigration algérienne s'est développée avec l'autonomisation grandissante des femmes : si 46 \% de la composante des flux en France est féminine, elles sont 32,6 \% dans les flux estudiantins et représentent $42,2 \%$ des entrées pour motif de travail. Ces derniers éléments montrent que la migration féminine se distingue progressivement de la migration familiale et prend son autonomie (figures 7 et 8). Il est possible d'affirmer que la crise économique doublée d'une crise politique profonde, marquant la transition du système politique algérien vers un système pluraliste favorisant l'intégration de la société algérienne dans ce qui est communément appelé "la mondialisation", semble avoir enclenché de nouvelles dynamiques migratoires.

\section{Des estimations difficiles}

L'Algérie est aujourd'hui un pays d'émigration. Il est cependant difficile de donner une estimation du nombre d'émigrés algériens sans accorder une attention particulière à la définition qui sous-tend les données statistiques publiées. En 2002, les services consulaires algériens ${ }^{(14)}$ évaluent les Algériens résidant à l'étranger à 1300000 (4,2 \% de la population algérienne), dont 1105000 résidant en France, soit $85 \%$ du total des migrants. Ces chiffres incluent les descendants de migrants qui sont nés dans le pays de résidence et qui n'ont, par conséquent, pas effectué de 


\section{Tableau 3 : Proportion des entrées d'Algériens en France}

\begin{tabular}{|c|c|c|c|}
\hline Année & $\begin{array}{l}\text { Entrées dans les pays } \\
\text { de l'OCDE }\end{array}$ & Entrées en France & $\begin{array}{c}\text { Proportion des entrées } \\
\text { en France }\end{array}$ \\
\hline 1995 & 12046 & 8362 & $69,4 \%$ \\
\hline 1996 & 12908 & 7833 & $60,7 \%$ \\
\hline 1997 & 17888 & 12221 & $68,3 \%$ \\
\hline 1998 & 22120 & 16747 & $75,7 \%$ \\
\hline 1999 & 18866 & 11396 & $60,4 \%$ \\
\hline 2000 & 23547 & 12419 & $52,7 \%$ \\
\hline 2001 & 28393 & 15028 & $52,9 \%$ \\
\hline 2002 & 35761 & 23446 & $65,6 \%$ \\
\hline 2003 & 39487 & 28548 & $72,3 \%$ \\
\hline 2004 & 43105 & 27934 & $64,8 \%$ \\
\hline 2005 & 38694 & 24755 & $64,0 \%$ \\
\hline 2006 & 41706 & 25412 & $60,9 \%$ \\
\hline 2007 & 39008 & 23107 & $59,2 \%$ \\
\hline 2008 & 37917 & 22315 & $58,9 \%$ \\
\hline 2009 & 35953 & 19961 & $55,5 \%$ \\
\hline
\end{tabular}

Source : OCDE

migration. L'OCDE évalue en 2005 à 1374634 les personnes nées en Algérie et immigrées dans les pays de l'OCDE, parmi eux 1257376 résident en France (91,5\%). Ce chiffre intègre les Français nés en Algérie. Si l'on prend en compte la définition française de l'immigré (né étranger à l'étranger), le nombre de migrants algériens en France n'est plus que de 691361 et le nombre total d'immigrés algériens dans les pays de l'OCDE en 2005 est dans ce cas de 858216 (tableau 2).

Le recensement rénové français estime pour l'année 2008 à 1366403 les personnes nées en Algérie, dont 714187 immigrés (nés étrangers à l'étranger), soit 52,3% (tableau 5). La proportion d'immigrants algériens en France n'est plus alors que de 80,6 \% du total des résidents dans les pays de l'OCDE. Comparée aux migrants tunisiens $(72,7 \%$ de résidents en France) et surtout marocains (31,4\%), on constate une plus faible diversification des pays de destination de l'émigration algérienne.

L'exploitation du recensement français (échantillon de 2008) permet de déterminer les caractéristiques socio-économiques des immigrés algériens en fonction de la période d'arrivée en France. La répartition de la population par tranches d'âge quinquennales 
(figure 9) a la forme d'une toupie, large au milieu et rétrécie à sa base et au sommet : elle est caractéristique d'une population qui continue d'être alimentée par un courant migratoire conséquent d'adultes des deux sexes. Au contraire, la représentation de la population des natifs d'Algérie considérés comme "non-immigrés" a la forme d'une pyramide inversée. Large au sommet et rétrécie à la base (figure 10), elle indique un ralentissement important de l'arrivée en France de cette catégorie de population.

La courbe retraçant les rapports de masculinité(15) selon les groupes d'âge quinquennaux a une allure classique pour les "non-immigrés" (plus de femmes que d'hommes dans les âges élevés) alors que celle des immigrés présente une allure différente avec une plus grande proportion de femmes entre 45 et 59 ans, quelle que soit la période d'arrivée en France, soit un rapport de masculinité inférieur à 100.

\section{Tableau 4 ; Introduction d'un conjoint sur le territoire français}

\begin{tabular}{|c|c|c|c|c|c|}
\hline Année & Conjoints & Masculin & Féminin & Total & $\begin{array}{l}\text { Total des } \\
\text { conjoints }\end{array}$ \\
\hline \multirow{2}{*}{2000} & d'étranger & 344 & 1439 & 1783 & \multirow{2}{*}{5265} \\
\hline & de Français & 1917 & 1565 & 3482 & \\
\hline \multirow{2}{*}{2001} & d'étranger & 489 & 1846 & 2335 & \multirow{2}{*}{7817} \\
\hline & de Français & 3109 & 2373 & 5482 & \\
\hline \multirow{2}{*}{2002} & d'étranger & 849 & 2594 & 3443 & \multirow{2}{*}{11255} \\
\hline & de Français & 4460 & 3352 & 7812 & \\
\hline \multirow{2}{*}{2003} & d'étranger & 1514 & 3277 & 4791 & \multirow{2}{*}{20968} \\
\hline & de Français & 9945 & 6232 & 16177 & \\
\hline \multirow{2}{*}{2004} & d'étranger & 1757 & 3596 & 5353 & \multirow{2}{*}{20350} \\
\hline & de Français & 8699 & 6298 & 14997 & \\
\hline \multirow{2}{*}{2005} & d'étranger & 1697 & 3587 & 5284 & \multirow{2}{*}{19527} \\
\hline & de Français & 8002 & 6241 & 14243 & \\
\hline \multirow{2}{*}{2006} & d'étranger & 303 & 1318 & 1621 & \multirow{2}{*}{15396} \\
\hline & de Français & 7336 & 6439 & 13775 & \\
\hline \multirow{2}{*}{2007} & d'étranger & 423 & 1989 & 2412 & \multirow{2}{*}{13403} \\
\hline & de Français & 5656 & 5335 & 10991 & \\
\hline \multirow{2}{*}{2008} & d'étranger & 493 & 2668 & 3161 & \multirow{2}{*}{12585} \\
\hline & de Français & 4839 & 4585 & 9424 & \\
\hline
\end{tabular}


Pour les groupes d'âge quinquennaux supérieurs à 60 ans, le rapport de masculinité est favorable à la gent masculine, proche de 200 (figure 11).

Le taux de chômage des immigrés nés en Algérie est relativement le plus élevé (28\%) ; il est 2,5 fois plus élevé que chez les personnes nées en Algérie "non immigrées" et presque 3 fois supérieur au taux de chômage global enregistré en France en 2008. Il est aussi supérieur à celui des immigrés nés au Maroc (25,1\%) ou en Tunisie (26,6\%). Les femmes sont dans une plus grande proportion que les hommes au chômage (32,8 \% contre 24,5 \%). Il faudrait cependant intégrer le fait qu'une personne de sexe féminin sur quatre est recensée comme femme au foyer. Enfin, sans surprise, la proportion de chômeurs est plus élevée dans les populations arrivées en France dans les périodes les plus récentes.

Le niveau d'instruction est plus élevé chez les immigrants arrivés en France dans les dernières décennies. Plus d'un immigré sur trois est titulaire du baccalauréat ou plus chez les personnes arrivées après 1998. Ils n'étaient que un sur cinq dans ce cas dans les années soixante-dix. Les différences de niveau d'instruction selon le sexe sont relativement minimes $(25,5 \%$ de titulaires du baccalauréat ou plus pour les hommes et $24,9 \%$ pour les femmes). Les proportions sont respectivement de 35,5 et 34,9\% pour les immigrés arrivés en France après 1998. Ces chiffres montrent que le départ des enseignants et intellectuels dans la décennie (1990) marquée par les violences a renforcé une tendance qui s'amorçait déjà et qui présente des similitudes avec les migrations marocaine et tunisienne orientées vers la France.

L'acquisition de la nationalité française par les immigrés nés (étrangers) en Algérie est dans une proportion de $42,1 \%$ (39,9\% pour le sexe masculin et 44,6 \% pour le sexe féminin) pour l'ensemble des recensés en 2008. Les immigrés arrivés en France entre 1989 et 1998 ont dans une plus forte proportion (58,1\%) acquis la nationalité française, soit $60 \%$ pour le sexe masculin et $57,1 \%$ pour le sexe féminin.

L'émigration algérienne a connu des variations tout le long du $\mathrm{XX}^{\mathrm{e}}$ siècle, évoluant d'une migration de travail temporaire vers une présence permanente en pays d'immigration. Contrairement à l'émigration marocaine qui a développé une diversification des pays d'arrivée depuis les années soixante-dix, elle s'est concentrée globalement en France et n'a connu de diversification substantielle vers les autres espaces d'immigration proches (Espagne, Italie) ou lointains (États-Unis, Canada) que tardivement. Malgré les mesures dissuasives et les dispositifs répressifs en Algérie et en France, les flux migratoires ont connu un regain de vitalité et une progression relativement importante ces dernières années. Les perspectives démographiques des deux rives de la Méditerranée ne laissent en aucune manière présager pour les années à venir un arrêt ou même un ralentissement de l'émigration du sud du Bassin méditerranéen vers l'Europe. 


\section{La migration vers l'Europe reste une solution possible et envisageable}

C'est ce que suggère Philippe Fargues ${ }^{(16)}$ en affirmant que les tendances actuelles (les effectifs nombreux de jeunes à l'entrée sur le marché du travail, l'accroissement de la population active féminine et l'élévation du niveau d'instruction au sein des nouvelles générations) favoriseraient la migration des populations du Maghreb vers l'Union européenne. La population active de l'UE diminuera en effet de près de 4 millions entre 2010 et 2015, de 5,6 millions entre 2015 et 2020, de 7 millions entre 2020 et 2025, et de 9,3 millions dans les cinq années qui suivront. Les projections des Nations unies prévoient, pour leur part, un solde migratoire négatif entre l'Algérie et le reste du monde de l'ordre de 28000 par an entre 2010 et 2020, de 18000 par an entre 2020 et 2030, et de 16000 par an entre 2030 et 2050. Soit, environ, un solde migratoire négatif de 780000 personnes entre 2010 et 2050. Ces projections prévoient pour la 
même période des soldes négatifs pour le Maroc et la Tunisie respectivement de l'ordre de 2,6 millions et de 160000 personnes.

Le potentiel migratoire gardera un niveau relativement élevé pendant les prochaines décennies. Car les effets de la baisse de la natalité et de la fécondité ne se feront sentir sur la fraction de la population ayant la plus forte propension à migrer qu'au-delà de 2020, date à laquelle arriveront sur le marché du travail les générations ayant des effectifs plus faibles. De ce fait, la concurrence pour les emplois disponibles sera rude pour tous les individus qui atteindront l'âge de 20 ans ou plus entre 2010-2025, période où les générations nées à la fin des années quatre-vingt et au début des années quatre-vingt-dix, générations ayant de gros effectifs de population, arriveront sur le marché du travail. Du fait de l'amorce tardive de la seconde phase de la transition démographique, les demandeurs d'emploi seront plus nombreux en Algérie qu'au Maroc, bien que les deux pays aient le même volume de population (figure 12). Avec l'hypothèse d'un taux de chômage à 10 \%, la demande d'emploi additionnelle 
est par conséquent de plus en plus importante. De 210000 par an au début des années quatre-vingt-dix, elle serait supérieure à 400000 par an pendant les périodes 20102015 et 2025-2030, et variera entre 250000 et 300000 par an jusqu'en 2040 (tableau 6). Ces calculs sont effectués avec les hypothèses d'un taux d'activité des femmes variant de $15 \%$ en début de période à 50 \% en fin de période et d'un taux de chômage global de $10 \%$ de la population active. Cette demande additionnelle s'ajoute naturellement au stock de demandeurs d'emploi déjà existant (environ 1,1 million en 2010). La demande additionnelle d'emploi ne diminuera d'une manière importante que lorsque les générations dotées de grands effectifs de population arriveront à l'âge de la retraite, c'est-à-dire à l'horizon de 2040. Tenant compte de ces perspectives d'emplois, il est fort possible que la migration continue à jouer le rôle d'atténuation des pressions sur le marché du travail local.

Fondamentalement, la composante des flux continuera d'évoluer dans le sens amorcé ces vingt dernières années, marquées par des changements substantiels dans le champ migratoire Maghreb-Europe aussi bien au niveau des pays de départ que d'arrivée.

\section{Tableau 5 : Répartition de la population selon le pays de naissance et la migration en France (2008)}

\begin{tabular}{|c|c|c|c|c|c|c|}
\hline \multirow{2}{*}{$\begin{array}{l}\text { Pays de } \\
\text { naissance et } \\
\text { situation à } \\
\text { l'immigration }\end{array}$} & \multicolumn{2}{|c|}{ Masculin } & \multicolumn{2}{|c|}{ Féminin } & \multicolumn{2}{|c|}{ Total } \\
\hline & Immigrés & $\begin{array}{c}\text { Non- } \\
\text { immigrés }\end{array}$ & Immigrés & $\begin{array}{c}\text { Non- } \\
\text { immigrés }\end{array}$ & Immigrés & $\begin{array}{c}\text { Non- } \\
\text { immigrés }\end{array}$ \\
\hline France & & 27475131 & & 29346171 & & 56821301 \\
\hline Portugal & 297178 & 10742 & 284536 & 11731 & 581715 & 22473 \\
\hline Italie & 155186 & 16740 & 161388 & 23603 & 316574 & 40343 \\
\hline Espagne & 114018 & 15298 & 144811 & 21620 & 258829 & 36917 \\
\hline $\begin{array}{l}\text { Autres États } \\
\text { membres } \\
\text { de I'UE }\end{array}$ & 282430 & 84670 & 372059 & 99833 & 654489 & 184502 \\
\hline Hors de I'UE & 98262 & 32807 & 124732 & 34686 & 222994 & 67493 \\
\hline Algérie & 378996 & 308137 & 335191 & 344079 & 714187 & 652216 \\
\hline Maroc & 344572 & 103124 & 311456 & 111542 & 656027 & 214666 \\
\hline Tunisie & 137213 & 64409 & 97501 & 71384 & 234714 & 135793 \\
\hline Autres & 824707 & 201864 & 883331 & 211415 & 1708038 & 413278 \\
\hline Total & 2632562 & 28312921 & 2715005 & 30276064 & 5347567 & 58588985 \\
\hline
\end{tabular}




\section{Tableau 6 : Projection des populations (active, en emploi et au chômage)}

\begin{tabular}{|c|c|c|c|c|c|c|c|}
\hline & \multirow[b]{2}{*}{ Masculin } & \multirow[b]{2}{*}{ Féminin } & \multirow[b]{2}{*}{ Total } & \multirow[b]{2}{*}{ Emploi } & \multicolumn{2}{|c|}{ Création d'emploi } & \multirow[b]{2}{*}{$\begin{array}{l}\text { Nombre de } \\
\text { chômeurs }\end{array}$} \\
\hline & & & & & $\begin{array}{l}\text { Quin- } \\
\text { quennale }\end{array}$ & Annuelle & \\
\hline 2010 & 9069440 & 1794000 & 10863440 & 9777096 & & & 1086344 \\
\hline 2015 & 10549600 & 2567200 & 13116800 & 11805120 & 2028024 & 405605 & 1311680 \\
\hline 2020 & 11198400 & 2727600 & 13926000 & 12533400 & 728280 & 145656 & 1392600 \\
\hline 2025 & 12638650 & 3622750 & 16261400 & 14635260 & 2101860 & 420372 & 1626140 \\
\hline 2030 & 13286350 & 4569300 & 17855650 & 16070085 & 1434825 & 286965 & 1785565 \\
\hline 2035 & 14597100 & 5526500 & 20123600 & 18111240 & 2041155 & 408231 & 2012360 \\
\hline 2040 & 14877000 & 6428400 & 21305400 & 19174860 & 1063620 & 212724 & 2130540 \\
\hline 2045 & 14846400 & 7209000 & 22055400 & 19849860 & 675000 & 135000 & 2205540 \\
\hline 2050 & 15360550 & 7840500 & 23201050 & 20880945 & 1031085 & 206217 & 2320105 \\
\hline
\end{tabular}

Sources calculées à partir des projections des Nations unies. Le taux d'activité féminine progresse de $15 \%$ à $50 \%$ en 2050 .

Concernant les pays de départ, le niveau d'instruction de la population s'est considérablement amélioré, l'urbanisation a connu une croissance accélérée. L'emploi dans les BTP s'est considérablement accru. En Algérie par exemple, il a été multiplié par 2,6 entre 2000 et 2008, passant de moins de 620000 à près de 1,6 million (enquêtes emplois, Office nationale des statistiques). Au niveau des pays d'arrivée, le développement de l'automatisation et la délocalisation des emplois industriels privilégiant la main-d'cuvre peu qualifiée, d'une part, et le vieillissement de la population, d'autre part, ont modifié les besoins du marché du travail. Les politiques de restriction et de sélection de l'immigration adoptées par les pays européens sont les conséquences de ces besoins d'ajustement.

Il en a résulté des changements considérables dans les profils migratoires. La première phase migratoire touche à sa fin (population rurale, analphabète et sans qualifications se dirigeant vers les emplois du BTP et de l'industrie en priorité), au profit d'une nouvelle vague migratoire qui a commencé à se dessiner au cours des années quatrevingt. Elle concerne prioritairement les populations urbaines scolarisées à différents degrés, une proportion de plus en plus importante de diplômés du supérieur, ainsi qu'un nombre de plus en plus grand de femmes prenant l'initiative de la migration et s'orientant vers les emplois tertiaires privilégiant les services aux entreprises et aux particuliers $^{(17)}$. 


\section{Notes}

1. Kamel Kateb participe aux travaux des unités de recherche "Migrations internationales et minorités" et "Identités et territoires des populations". Ses travaux portent sur l'histoire statistique de l'Algérie, sur l'histoire des populations des pays du Maghreb, sur les systèmes éducatifs de ces pays et sur les migrations entre le Maghreb et l'Europe.

2. Kamel Kateb, Européens, "indigènes" et juifs en Algérie (1830-1962), Paris, INED/PUF, 2001.

3. Selon la définition retenue par l'OCDE, il le serait par plus de 4 pendant la même période. Les données statistiques concernant les migrations sont les plus sujettes à contestation; elles dépendent des définitions adoptées. L'OCDE définit les migrants comme des personnes résidant depuis plus d'un an dans un pays différent de leur pays de naissance; alors que la France, par exemple, définit un migrant comme une personne "née étrangère dans un pays étranger". L'adoption de telle ou telle définition se répercute significativement sur l'estimation du nombre de migrants. Concernant la définition de l'OCDE, elle inclut parmi les migrants d'Algérie toutes les personnes rapatriées d'Algérie et nées dans ce pays. La différence avec la définition française est de plus de 600000 personnes. Ce n'est pas la seule difficulté dans le dénombrement des migrants. Les pays sans registre de population ne tiennent que la comptabilité des entrées sur leur territoire et ne tiennent pas compte des sorties et de la migration de retour. La fiabilité des chiffres qu'ils communiquent est par conséquent relativement sujette à caution.

4. Kamel Kateb, "La gestion administrative de l'émigration algérienne vers les pays musulmans au lendemain de la conquête de l'Algérie (1830 à 1914)", in Population, n², avril 1997, pp. 399-428.

5. Kamel Kateb, Européens, "indigènes” et juifs en Algérie (1830-1962), op. cit.

6. Kamel Kateb, "La gestion administrative de l'émigration algérienne vers les pays musulmans au lendemain de la conquête de l'Algérie (1830 à 1914)", art. cit.

7. Xavier Yacono, Histoire de l'Algérie depuis la régence, Versailles, L'Atlanthrope, 1993.

8. Kamel Kateb, Européens, "indigènes” et juifs en Algérie (1830-1962), op. cit.

9. Ibid.

10. Recensement de 1966.

11. Les pouvoirs publics en Algérie n'appréhendent l'émigration algérienne que sous l'angle de son contrôle politique. Contrairement à la Tunisie et au Maroc, les transferts des migrants ne jouent pas un rôle essentiel dans les équilibres des balances de paiement. Les ressources provenant du pétrole assurent les besoins en financement de l'économie. De ce fait, les pouvoirs publics n'accordent un intérêt particulier aux migrants que pendant les campagnes électorales. La politique suivie est pratiquement définie par les accords signés avec le gouvernement français ou dans le cadre du processus de Barcelone (5+5). Il s'agit essentiellement du blocage de la migration clandestine.

12. Les données du ministère de l'Intérieur (source $A G D R E F)$ permettent de déterminer le nombre d'étrangers entrés majeurs en France et obtenant, pour la première fois, un titre de séjour d'une durée de validité supérieure ou égale à un an, et le nombre d'entrées de mineurs issus de ressortissantes d'un pays de l'Espace économique européen ou de mères réfugiées (il s'agit d'une estimation afin de pallier l'absence d'enregistrement de ces deux types de flux).

13. Agence des Nations unies pour les réfugiés (UNHCR), Statistical Year book, 2001, Genève, Nations unies, 2002.

14. Anna Di Bartolomeo, Thibaut Jaulin et Delphine Perrin, "CARIM - Migration Profile, Algérie", rapport écrit sur la base des données et des publications du CARIM, Consortium for Applied Research on International Migration.

15. Le rapport de masculinité consiste à calculer le nombre d'hommes pour 100 femmes, il permet de détecter des incohérences dans une population ou de révéler des situations anormales. Par exemple, à la naissance, il y a 105 enfants de sexe masculin pour 100 enfants de sexe féminin. Dans les pays où ce rapport avoisine les 120 , on en déduit qu'il y a une sélection selon le sexe. Autre exemple, lorsque dans un pays il y a plus de femmes que d'hommes entre 20 et 40 ans, on en déduit de forts mouvements migratoires d'hommes seuls. Aux âges élevés, sans migration, il y a plus de femmes que d'hommes, donc un rapport de masculinité inférieur à 100 .

16. Philippe Fargues, "Emerging demographic patterns across the Mediterranean and their implications for migration through 2030", Migration Policy Institute, novembre 2008, www.migrationpolicy.org/pubs/FarguesPaper.pdf

17. Pour le Maroc la première phase connaît un prolongement et coexiste probablement avec la seconde, notamment à la faveur de la migration vers l'Espagne et l'Italie (besoin de main-d'ceuvre dans l'agriculture). Ce qui explique l'importance des flux marocains comparativement à l'Algérie et à la Tunisie. 